\title{
INFLUENCE DE LA DIFFUSION MULTIPLE DANS LE CONTRÔLE ULTRASONORE DES ACIERS AUSTÉNITIQUES À GROS GRAINS
}

\author{
J.M. BORDIER, M. FINK, A. LE BRUN* et F. COHEN TENOUDJ** \\ LOA/ESPCI, 10 rue Vauquelin, F-75005 Paris, France \\ *EDF/DER, 25 allée Privée, Carrefour Pleyel, F-93206 Saint-Denis, France \\ ** LUAP, Université Paris 7, Tour 23, 2 place Jussieu, F-75005 Paris, France
}

\section{RESUME}

Pour améliorer la réduction de bruit acoustique dans les aciers austénitiques à gros grains, nous avons soigneusernent étudié les propriétés statistiques et spectrales des champs ultrasonores rétrodiffusés et transmis. Ces propriétés sont très différentes de celles observées dans l'étude des milieux biologiques : la fonction de corrélation spatiale du champ de pression diffusé (mesurée à l'aide d'une barrette multi-éléments) est très étroite et la fréquence centrale du bruit diffusé augmente avec le temps. Ces résultats ne peuvent pas être expliqués par un processus de diffusion simple sur les grains austénitiques : leur explication nécessite un processus de diffusion multiple.

\section{ABSTRACT}

To optimize the acoustic noise reduction from large grains austenitic steel, we have carefully studied the statistical and spectral behaviour of the backscattered and transmitted ultrasonic fields. Compared to the results obtained in biological tissues, the behaviour of these wavefields is drastically different : the spatial correlation fonction of the scattered pressure field (measured with multi-elements) is very narrow, while the central frequency of the scattered noise increases with time. These results cannot be explained with single scattering processes on the austenitic grains, multiple scattering processes are necessary to explain this particular behaviour

\section{INTRODUCTION}

Jusqu'à aujourd'hui, le contrôle ultrasonore des aciers austénitiques moulés statiques (grains de l'ordre du mm) s'est révélé à la fois peu fiable et peu performant. La raison principale en incombe à un important bruit de grains acoustique dû à l'intéraction des ondes ultrasonores avec la structure interne de ces aciers. L'onde incidente subit un processus de diffusion sur les joints de grains qui est directement relié à la taille et à l'orientation de ces grains par rapport à la direction de propagation [1]. Chaque tranche d'acier austénitique se comporte, après insonification, comme une source de champ acoustique diffusé. Le caractère aléatoire de la répartition des grains permet d'assimiler ce type de source à une source incohérente. L'apparition, tout au cours de la propagation, de ce champ diffusé qui peut devenir plus important que le champ incident non perturbé, entraine une perte de cohérence du champ ultrasonore. Cette perte de cohérence entraine une distorsion des fronts d'onde qui se manifeste par une défocalisation importante. Le repérage des défauts au moyen de faisceaux défocalisés se trouve aussi compliqué, par la présence du bruit acoustique rétrodiffusé qui masque souvent l'écho des défauts. Le rapport d'amplitude entre le signal échographique provenant des défauts et le bruit acoustique est souvent très faible [2].

Dans un papier récent [3], nous avons présenté l'intérêt des transducteurs à phase aléatoire dans l'amélioration du rapport signal à bruit. Pour prévoir les performances de ces transducteurs, nous avons introduit le concept de grains d'informations décorrelés qui permet de prédire l'amélioration du rapport signal à bruit. Nous avons montré que l'efficacité d'un traitement incohérent de l'information dépend du nombre d'informations décorrélées, provenant du matériau, que l'on peut capter sur l'ouverture de réception.

Nous nous proposons, ici, d'étudier expérimentalement les propriétés statistiques et spectrales des champs ultrasonores rétrodiffusés et transmis par ces aciers à l'aide des mesures des fonctions de corrélation spatiale des champs et de la mesure de l'évolution temporelle de la fréquence centrale des signaux. Nous mettons l'accent sur les différences significatives, de résultats obtenus par ailleurs, avec les matériaux diffuseurs biologiques. Nous en déduisons l'importance de la diffusion multiple dans la propagation au sein des aciers austénitiques lorsqu'on travaille avec des fréquences de quelques $\mathrm{MHz}(>2 \mathrm{MHz}$ ). Une autre approche pour qualifier la diffusion multiple consiste à travailler en transmission. On observe alors, après la traversée de l'acier, un premier front cohérent suivi d'un bruit incohérent qui dure longtemps et qui correspond à la diffusion multiple sur des trajets de plus en plus longs.

\section{1/ ARGUMENT THEORIQUE}

Toute cette étude est basée sur l'utilisation du théorême de Van Cittert Zernike qui est utilisé en optique moderne [4]. Ce théorême va nous permettre de remonter à la taille de la zone qui diffuse effectivement le champ ultrasonore et de recueillir des informations sur la nature de la diffusion de l'onde incidente. 


\section{1 - LE THEOREME DE VAN CITTERT ZERNIKE APPLIQUE A L'ACOUSTIQUE}

Le théorême de Van Cittert Zernike décrit la propagation de la fonction de corrélation spatiale d'un champ ondulatoire produit par une source incohérente. Il nous apprend que la fonction de corrélation spatiale du champ, émis par cette source, est la transformée de Fourier de la fonction d'ouverture de cette source.

Dans le domaine de l'échographie ultrasonore, la zone incohérente n'est pas le transducteur d'illumination (il est cohérent), mais la zone du matériau qui rétrodiffuse la pression incidente. C'est en mesurant la fonction de corrélation spatiale du champ rétrodiffusé que l'on peut évaluer l'expansion de cette zone de réémission. Le théorême de Van Cittert Zernike nous apprend en effet que plus la zone qui donne naissance au phénomène de diffusion est étroite, plus la fonction de corrélation spatiale est large.

\section{2 - LA DIFFUSION MULTIPLE}

D'une façon générale, la diffusion se produit dans les milieux de propagation inhomogène ou la densité et l'élasticité sont définies en chaque point de l'espace. Les variations de densité et d'élasticité traduisent les passages sur les défauts mais aussi les inhomogénéités se trouvant à l'intérieur des milieux (les grains dans les aciers).

Dans les milieux faiblement inhomogènes, on utilise l'approximation de Born du premier ordre qui revient à négliger la diffusion multiple. On néglige le fait qu'une onde diffusée sur une hétérogénéité puisse être, plus tard, diffusée par les autres hétérogénéités.

L'hypothèse de Born du premier ordre peut être remise en cause dans un milieu ou les diffuseurs possèdent une section efficace de diffusion importante. Lorsque l'intéraction entre les diffuseurs est prise en compte, chaque élément de volume du domaine diffusant voit une superposition de l'onde incidente et des ondes diffusées par les regions avoisinantes de sorte que l'onde effectivement reçue par un élément de volume possède une phase différente de la phase de l'onde initiale incidente. A mesure que la distance de propagation grandit, l'énergie de l'onde incidente diminue relativement à l'énergie de l'onde diffusée. A partir d'une certaine distance, l'intensité incohérente, qui provient du diffusé, dépasse l'intensité cohérente provenant de l'onde incidente.

\section{2/ RESULTATS EXPERIMENTAUX PAR MODE ECHOGRAPHIQUE}

Dans un premier temps, nous avons mesuré la cohérence spatiale du champ de pression rétrodiffusé par les matériaux. Pour cela, il s'avère nécessaire d'échantillonner spatialement le champ de pression par un ensemble de transducteurs de petites dimensions. Comme les transducteurs cohérents employés habituellement présentent des dimensions trop grandes relativement aux fluctuations du champ de pression ultrasonore, nous avons utilisé une barrette linéaire qui possède des éléments de petite taille. Le signal reçu par chaque élément est une transcription fidèle de la pression au point correspondant (à une convolution temporelle par la réponse acousto-électrique près).

Dans un deuxième temps, nous avons analysé les signaux échographiques reçus sur chacun des éléments de la barrette en mesurant l'évolution de la fréquence centrale des signaux en fonction du temps.

\section{1 - DESCRIPTION DU MONTAGE EXPERIMENTAL}

La barrette de transducteurs utilisée compte 64 éléments piézoélectriques, distants de $0.75 \mathrm{~mm}$, dont les largeurs et hauteurs sont respectivement de $0.6 \mathrm{~mm}$ et $10 \mathrm{~mm}$. Sa fréquence centrale est de $3 \mathrm{MHz}$.

La barrette est immergée dans l'eau. Elle insonifie un échantillon d'acier, d'épaisseur $80 \mathrm{~mm}$, qui possède une structure à gros grains équiaxes de taille moyenne $5 \mathrm{~mm}$. Le signal acoustique rétrodiffusé est alors capté sur les 64 transducteurs élémentaires. L'émission est focalisée électroniquement, au moyen d'une loi de retard, à $40 \mathrm{~mm}$ de profondeur dans l'acier. Les 64 signaux de pression sont captés et stockés numériquement (figure $n^{\circ} 1$ ).

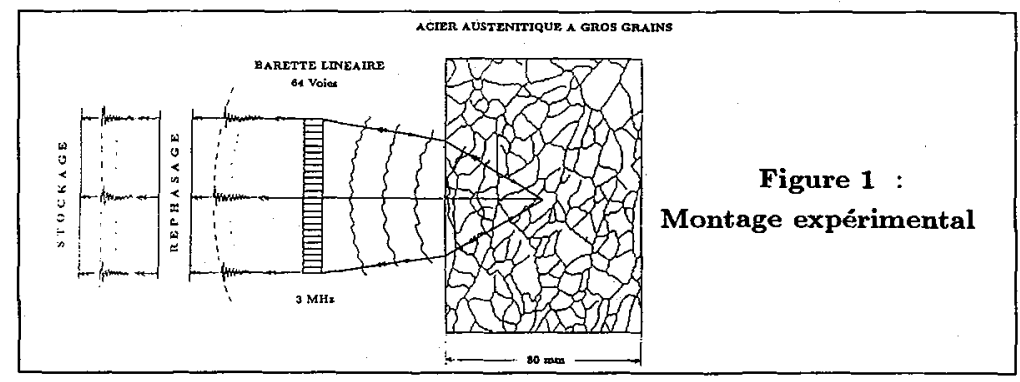

\section{2 - MESURE DE LA COHERENCE SPATIALE DU CHAMP RETRODIFFUSE}

Pour mesurer la cohérence spatiale du champ rétrodiffusé, nous avons calculé sa fonction de corrélation spatiale puis nous avons analysé cette fonction à travers le théorême de Van Cittert Zernike. On verra que, pour une émission focalisée dans les aciers austénitiques, la taille réelle de la zone d'acier insonifiée est beaucoup plus grande que celle prévue par la théorie de la diffraction du faisceau focalisé.

2.2.1 - Estimation de la fonction de covariance spatiale du champ de pression rétrodiffusé

L'échantillonnage spatial et temporel du champ de pression par une barrette linéaire nous donne un signal bidimensionnel fonction du temps et de l'espace. Chaque transducteur élémentaire de rang i délivre un signal proportionnel à la pression $p(i, t)$. La variable $i$ (repérant le centre du transducteur considéré) correspond à la variable spatiale. De son coté la variable t est reliée à la profondeur de la zone explorée.

En sélectionnant, autour du temps d'observation t une tranche de signal de pression au moyen d'une porte temporelle de durée $T$, nous pouvons évaluer une fonction de corrélation spatiale normalisée en fonction de la distance.entre deux 
transducteurs de la barrette linéaire. Cette distance, qui est mesurée par le nombre de pas de la barrette séparant les deux transducteurs considérés, est représentée par la valeur $n$. n peut donc varier de 1 à $N$, ou $N$ est le nombre total des éléments de la barrette. La variance de la fonction de corrélation peut être réduite en moyennant le calcul sur tous les couples de transducteurs séparés d'une même distance de n pas. On obtient ainsi un estimateur de la fonction de corrélation spatiale du signal échographique provenant d'une tranche de signal située au temps $t$.

$$
\hat{R}_{p}(n, t)=\frac{N}{N-n} \frac{\sum_{i=1}^{N-n} \int_{t-\frac{T}{2}}^{t+\frac{T}{2}} p(i, t) p(i+n, t) d t}{\sum_{i=1}^{N} \int_{t-\frac{T}{2}}^{t+\frac{T}{2}} p^{2}(i, t) d t}
$$

Dans nos expériences, nous utilisons des fenêtres de largeur $\mathbf{T}=2 \mu \mathrm{s}$. Dans le cas d'une émission focalisée de distance focale $F$, nous pouvons sélectionner la pression rétrodiffusée par la zone focale en centrant la fenêtre au temps focal. Il faut noter qu'un recalage des signaux en temps, qui tienne compte de la courbure de focalisation incidente, est nécessaire.

\subsection{3 - Mesure de la covariance spatiale du champ rétrodiffusé}

La courbe $n^{\circ} 1$ représente la fonction de corrélation spatiale du champ rétrodiffusé par la zone située à $40 \mathrm{~mm}$ de profondeur dans l'acier. Nous remarquons que cette fonction est très étroite. Le signal acoustique rétrodiffusé est donc très vite décorrélé, c'est à dire que des zones très voisines situées sur la barrette reçoivent des signaux très dissemblables, bien que le faisceau incident soit théoriquement focalisé sur une petite région du matériau. Un tel résultat ne peut s'expliquer que par un élargissement de la zone diffusante (voir 1.1) par un processus de diffusion multiple qui élargit très fortement la tache focale, réalisant un véritable "halo ultrasonore" autour du faisceau incident. C'est de ce halo que provient le signal diffusé.

Dans le cas de milieux diffuseurs biologiques [5], l'approximation de Born du premier ordre est bien vérifiée et le faisceau incident n'est pas défocalisé. Les diffuseurs sont petits devant la longueur d'onde; leur section efficace de diffusion est faible et l'hypothèse d'un milieu incohérent est valable. On observe alors une fonction de corrélation spatiale très large qui a bien l'allure d'une fonction triangle prévue par le théorême de Van Cittert Zernike.

\section{3 - ANALYSE DES SIGNAUX ECHOGRAPHIQUES}

Dans le but de préciser le comportement particulier de cet acier, nous nous sommes attachés à caractériser l'évolution temporelle de la fréquence centrale des 64 signaux échographiques rétrodiffusés.

\subsection{1 - Estimation de l'évolution temporelle de la fréquence centrale des signaux échographiques}

Dans un premier temps, nous échantillonnons le signal à étudier à l'aide d'une fenêtre temporelle glissante de durée constante. Puis, à l'intérieur de chaque fenêtre ainsi définie nous calculons le spectre de puissance. Si on considère la vitesse du son comme constante, chaque fenêtre isolant la réponse échographique d'une tranche de milieu comportant des diffuseurs, on repère l'information en provenance d'un domaine restreint du matériau dans le sens de la profondeur. Le spectre d'une fenêtre est chahuté puisqu'il correspond à plusieurs échos superposés, chaque écho provenant d'un diffuseur différent. Néanmoins, sous l'effet de l'atténuation de l'onde, on s'attend classiquement à observer un décalage du contenu spectral vers les basses fréquences lorsque les fenêtres correspondent à des échos de diffuseurs de plus en plus profonds.

\subsection{2 - Mesure de l'évolution temporelle de la fréquence centrale des signaux échographiques}

Les signaux échographiques reçus par les 64 éléments de la barrette sont découpés par une suite de fenêtres de Hanning glissantes, d'une largeur de $5.12 \mu \mathrm{s}$, puis analysés dans le domaine de Fourier. La courbe $\mathrm{n}^{\circ} 2$ montre l'évolution temporelle de la position des centres de gravité fréquentiels de spectre, moyennée sur les 64 signaux. Nous remarquons que contrairement à ce que l'on obtient dans l'étude d'un milieu diffuseur biologique ou la fréquence centrale diminue avec la propagation de l'onde on observe ici un sensible accroissement de cette frequence centrale.

L'hypothèse sur la diffusion multiple aux joints de grains nous permet de fournir une explication à ce léger accroissement de la fréquence. Chaque diffusion permet au signal de s'enrichir en hautes fréquences (voir 1.2) dans la mesure ou nous travaillons dans une gamme de fréquences ou la section efficace de diffusion croit avec la fréquence. Le nombre des diffusions multiples est tel qu'il fait plus que compenser l'effet de l'absorption thermique qui défavorise le contenu spectral hautes fréquences avec la propagation.

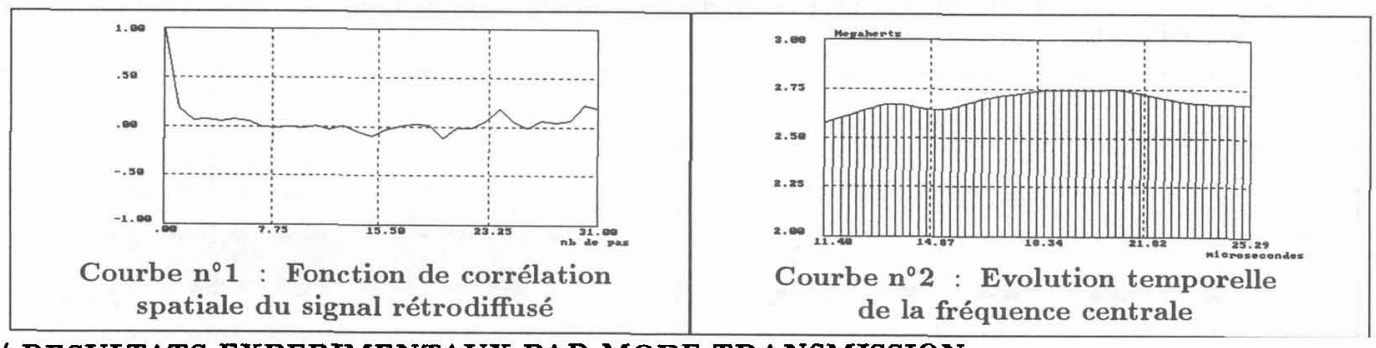

\section{3/ RESULTATS EXPERIMENTAUX PAR MODE TRANSMISSION}

Pour valider l'hypothèse de diffusion multiple de l'onde aux joints de grains, nous avons, en plus des expériences effectuées par échographie, mesuré le champ ultrasonore transmis à travers l'échantillon d'acier.

Nous mesurons, notamment, la fonction de covariance spatiale ainsi que l'évolution temporelle de la fréquence centrale des signaux transmis. Les paramètres de calcul utilisés lors de ces calculs sont les mêrnes que ceux employés lors des expériences en échographie. Nous mettons eri évidence la dualité physique de l'onde transmise qui est constituée d'une onde de nature cohérente et d'une autre de nature incohérente. 


\section{1 - DESCRIPTION DE L'EXPERIENCE}

La barrette linéaire est conservée en émission. Le signal transmis à travers l'acier est recueilli sur un hydrophone qui est déplacé parallèlement à la face arrière de l'échantillon. Le faiscean d'émission est focalisé sur l'hydrophone comme le montre la figure 2. L'hydrophone est déplacé suivant un pas de $0.5 \mathrm{~mm}$ de part et d'autre de l'axe focal sur une distance de $10 \mathrm{~mm}$.

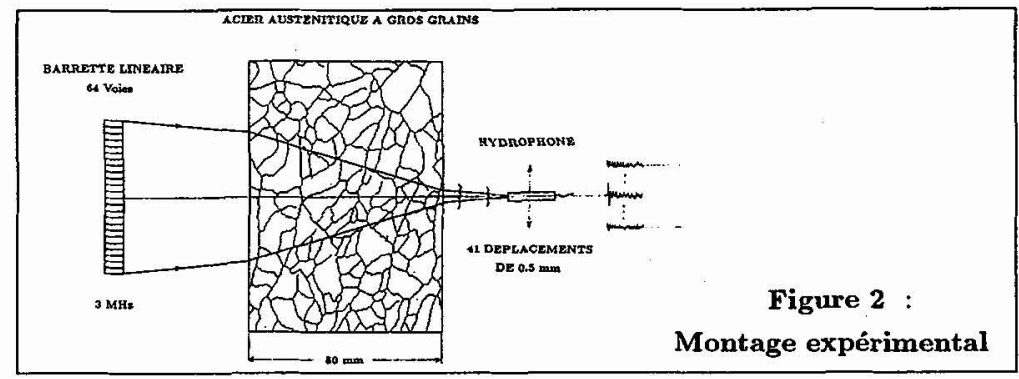

\section{2 - ALLURE DU SIGNAL ULTRASONORE TRANSMIS}

Les signaux transmis présentent un premier front de moins en moins marqué à mesure que l'hydrophone s'éloigne du point focal, puis un train d'onde de longue durée dont l'amplitude tend à croitre avec le temps. Sur la courbe $\mathrm{n}^{\circ} 3$, qui représente la moyenne des enveloppes des signaux transmis, sur toutes les positions de l'hydrophone, les deux composantes de cette onde sont clairement distinctes. On y observe l'arrivée directe d'un front d'onde qui représente la partie cohérente du signal et ensuite l'arrivée d'une onde de pression importante, de type aléatoire, qui dure très longtemps et qui provient manifestement de tous les chemins acoustiques qui ne sont pas en ligne droite et qui correspondent à des diffusions multiples. L'accroissement de l'amplitude du signal avec le temps s'explique par la multiplication des chemins acoustiques à travers l'échantillon à mesure que le temps croit. Le volume décrit par l'ensemble des chemins acoustiques augmente donc avec le temps tout comme l'énergie.

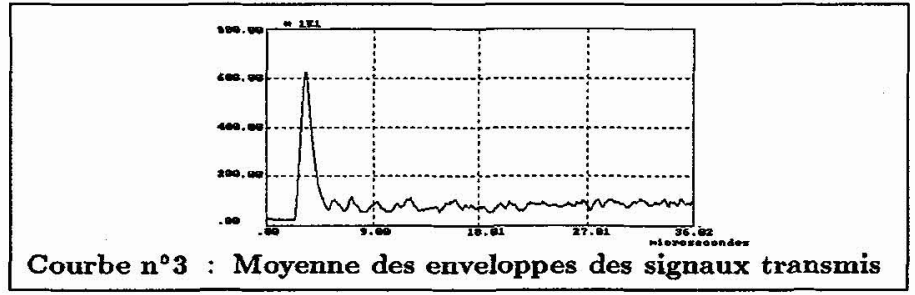

\section{3 - ANALYSE DU SIGNAL INCOHERENT}

3.3.1 - Mesure de la fonction de covariance spatiale

L'étude de la covariance spatiale du bruit transmis de cette composante incohérente montre, de la même façon qu'en échographie, que la covariance spatiale est très étroite (courbe $\mathrm{n}^{\circ} 4$ ). Le bruit acoustique provient, en fait, $\mathrm{d}^{\mathbf{u} u n e} \mathrm{zone}$ beaucoup plus grande que celle définie par la largeur du faisceau cohérent.

\subsection{2 - Mesure de la fréquence centrale des signaux}

La courbe $\mathrm{n}^{\circ} 5$ montre l'accroissement de la fréquence centrale du signal en fonction du temps. De nouveau, ce résultat ne peut s'interpréter qu'à travers un phénomène de diffusion multiple favorisant les fréquences élevées du signal à chaque diffusion.

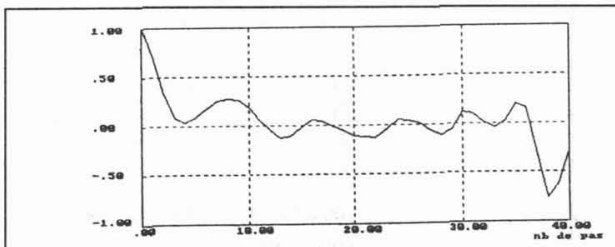

Courbe $n^{\circ} 4$ : Fonction de corrélation spatiale du signal transmis

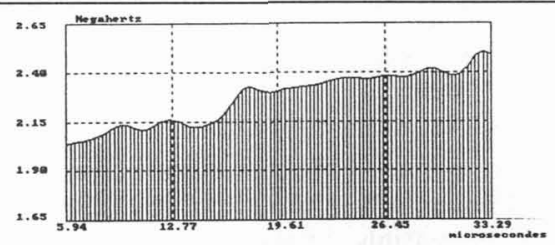

Courbe $n^{\circ} 5$ : Evolution temporelle de la fréquence centrale 


\section{CONCLUSION}

Du fait des hétérogénéités acoustiques que représentent les joints de grains, les faisceaux ultrasonores subissent des phénomènes de diffusion multiple qui se manifestent d'une part par une distorsion très forte des fronts d'onde impulsionnels ultrasonores qui perdent au cours de leur propagation leur caractère de surface d'onde pour devenir de véritables volumes d'onde ou se mélangent l'onde incidente, qualifiée d'onde cohérente, et les ondes diffusées une ou plusieurs fois aux joints de grains qui forment la composante incohérente du signal.

En mode échographique, le signal rétrodiffusé par le milieu n'est lié qu'à la partie incohérente du signal. C'est ce signal qui se manifeste sous la forme d'un bruit échographique très génant pour repérer un défaut.

Dans l'étude que nous avons faite, nous avons mis au point une méthode de caractérisation de la partie incohérente du signal que nous avons séparée clairement de la partie cohérente du signal.

Nous avons mis en évidence, à travers des mesures de la fonction de corrélation spatiale des signaux réféchis ou transmis par ces aciers, l'incohérence spatiale totale des champs de pression correspondants. Cette propriété particulière des aciers contribue à l'obtention d'un nombre important de données décorrélées sur les ouvertures de réception ce qui augure bien du grand potentiel de réduction de rapport signal à bruit des signaux ultrasonores recueillis à partir des aciers austénitiques à gros grains sur des transducteurs de type incohérent.

\section{REFERENCES}

[1] Truell, Elbaum et Chick. "Ultrasonic Methods in Solid State Physics". Acad. Pr., New-York - London, 1969.

[2] "Ultrasonic Characterization of Centrifugally Cast Stainless Steel". Palo Alto, CA: Electric Power Research Institute, June 1987, NP-5286.

[3] M. Fink, R. Mallart et F. Cancre. IEEE Trans. Ultras. Ferroelec. Freq. Control. Vol 37, 1990.

[4] J.W. Goodman, Statistical Optics. J. Willey \& sons, Inc. New-York 1985, chap. 5.

[5] R. Mallart et M. Fink. "The Van Cittert-Zernike theorem in pulse echo measurements". J. Acoust. Soc. Am. 